scope of outcomes studied, and evaluate for methodological issues.

Methods With an academic librarian, we developed search strategies in MEDLINE and Embase (January 1990 - May 2017) to search for full-length English articles. Our strategies were iteratively fine-tuned and finalized after peer-review by librarians. We supplemented the search with hand-searching of the references of review articles and included articles. If a study included both pediatric and adult subjects, we included the study only if the median or mean age at outcome ascertainment was $\geq 18$ years. Information about outcomes and study designs was recorded. All studies were graded independently by two reviewers using the Quality in Prognosis Studies risk-of-bias tool.

Results Of 14100 articles, 26 publications were included in this review. Of these, only 10 (36\%) focused on adults; while the rest studied mixed adult and paediatric populations. Two studies $(7 \%)$ were longitudinal (repeat measures on $\geq 3$ occations) while the remainder assessed outcomes on a single occasion. About $80 \%$ of studies were published within 20102017. The most commonly studied diseases were SLE (61\%), IIM (18\%), and systemic sclerosis (11\%). The most commonly reported primary outcomes were organ damage (29\%), cardiovascular outcomes (14\%), and mortality (14\%). The mean ages at outcome assessment were 19.5-46.8 years for adultonly studies and 19.3-35 years for mixed studies. Moderate to high risk-of-bias was found in all studies for study participation, $90 \%$ for study attrition, $61 \%$ for prognostic factor measurement, $36 \%$ for outcome measurement, $89 \%$ for confounding, and 54\% for statistical analysis.

Conclusion There is need for more information about adulthood outcomes of ChildSARDs. Longitudinal data was especially lacking. We recommend that future studies on ChildSARD outcomes be undertaken in the framework of a longitudinal cohort. Adult outcomes should be separately reported from pediatric outcomes in a mixed cohort. Study populations should be clearly defined to allow for accurate MESH coding so as to facilitate easy searching for such information and for knowledge dissemination. Careful attention should be paid during study design to reduce bias in choice of study populations, especially in accounting for attrition and confounding.

\section{CS-22 CONFIRMATORY FACTOR ANALYSIS OF THE PATIENT- REPORTED PERCEIVED DEFICITS QUESTIONNAIRE IN SYSTEMIC LUPUS ERYTHEMATOUS: CAUTIONS FOR USE OF SUBSCALES}

${ }^{1}$ Lisa Engel ${ }^{*},{ }^{1}$ Jiandong Su, ${ }^{2}$ Emily Nalder, ${ }^{3}$ Yael Goverover, ${ }^{1,2,4}$ Monique Gignac, ${ }^{1,2}$ Carmela Tartaglia, 'Nicole Anderson, 1,2Zahi Touma. 'University Health Network, Toronto, ON, Canada; ${ }^{2}$ University of Toronto, Toronto, ON, Canada; ${ }^{3}$ New York University, New York, NY, USA; ${ }^{4}$ Institute for Work and Health, Toronto, ON, Canada

\subsection{6/lupus-2018-Ism.57}

Background Approximately 38\% of adults living with Systemic Lupus Erythematosus (SLE) experience cognitive impairment (CI) that can detrimentally affect employment, disease selfmanagement, and quality of life. Identifying those with SLE related CI is critical, but is difficult to do in busy and resource-limited clinics. The patient-reported 20-item Perceived Deficits Questionnaire (PDQ-20), used to screen for SLE related $\mathrm{CI}$, could be less time and cost-burdensome than other objective instruments. However, there is a dearth of published measurement property evidence for using the PDQ-20 with SLE patients. In adults with Multiple Sclerosis the PDQ-20 is purported to have four factors (subscales): attention/concentration, retrospective memory, prospective memory, and planning/ organization. This structure has not been examined in adults with SLE. The purpose of this study is to examine the factor structure and the internal consistency of the PDQ-20 in an SLE cohort.

Methods Consecutive SLE patients aged 18-65 years were recruited from a single rheumatology center between July 2016 and March 2018. Patients completed the PDQ-20. Analyses included socio-demographic descriptive analyses and confirmatory factor analyses (CFA) of the purported PDQ-20 four-factor structure. Sample size calculations indicated that a cohort of $n=177$ was sufficient to perform the CFA (power $=0.99$ ). Analysis was completed on returned baseline PDQ-20 data using SAS ${ }^{\circledR}$ software.

Results Patient demographics are presented in table 1. There was no missing PDQ-20 data. CFA model fitting was adequate (standardized root mean square residual $=0.05$; root mean square error of approximation $=0.10$; Bentler comparative fit index $=0.90$ ). All factor loadings were statistically significant (factor loading range 0.55-0.88; all t-value >9.82). All factors highly correlated with each other (correlation range: $0.87-$ 0.97; all $\mathrm{p}<0.01)$. Lagrange Multiplier (LM) tests indicated that multiple alternate item-factor pathways could improve the four-factor model (ten largest significant LM statistics range from 7.92-20.78; new possible pathways for 7 items to other factors). Item 19 ('forget to take medication') had low reliability to its purported factor ('prospective memory'; $\mathrm{R}^{2}=0.30$ ). The internal consistency (Cronbach's alpha) for the four factors ranged from 0.82 to 0.91 .

Abtract CS-22 Table 1 Socio-demographic information of recruited patients $(n=208)$ who returned and did not return PDQ-20*

\begin{tabular}{|c|c|c|}
\hline & $\begin{array}{l}\text { PDQ-20 returned } \\
(n=177)\end{array}$ & $\begin{array}{l}\text { PDQ-20 not } \\
\text { returned } \\
(n=31)\end{array}$ \\
\hline \multicolumn{2}{|l|}{ (\% female) } & $83.9 \%$ \\
\hline \multicolumn{2}{|l|}{ (mean $\pm S D$ years) } & $41.97 \pm 12.75$ \\
\hline Age at SLE diagnosis (mean \pm SD years) & $28.16 \pm 10.48$ & $28.57 \pm 10.19$ \\
\hline $\begin{array}{l}\text { Highest education level obtained at } \\
\text { study visit }\end{array}$ & $\begin{array}{l}\text { High-school or less } \\
25.1 \%\end{array}$ & $\begin{array}{l}\text { High-school or less } \\
16.1 \%\end{array}$ \\
\hline (\% of sample ${ }^{\text {I) }}$ & $\begin{array}{l}\text { College/University } \\
74.9 \%\end{array}$ & $\begin{array}{l}\text { College/University } \\
83.9 \%\end{array}$ \\
\hline Employment status at study visit & Employed $51.7 \%$ & Employed $73.3 \%$ \\
\hline \multirow[t]{8}{*}{ (\% of sample ${ }^{2}$ ) } & Retired $2.9 \%$ & Retired $0.0 \%$ \\
\hline & Homemaker $6.9 \%$ & Homemaker $3.3 \%$ \\
\hline & Student $5.2 \%$ & Student $6.7 \%$ \\
\hline & Disability/sick leave & Disability/sick leave \\
\hline & $27.6 \%$ & $16.7 \%$ \\
\hline & Looking for work & Looking for work \\
\hline & $4.0 \%$ & $0.0 \%$ \\
\hline & Other $1.7 \%$ & Other $0.0 \%$ \\
\hline
\end{tabular}

*All demographic variables not statistically significantly different between groups ( $p>0.05$ ); all data from baseline visits of longitudinal study.

${ }^{\text {n }} \mathrm{n}=170$ (7 patients missing data) for returned group; $n=31$ for not returned

${ }_{n}=174$ (3 patient missing data) for returned; $n=30$ ( 1 missing) for not returned 
Conclusions The CFA analyses indicate that while the fit of the four-factor model for the PDQ fits, the model could be improved. Particularly concerning is the different factor-pathways for seven items, item 19's current low item-factor reliability, and the increased correlations between factors. In adult SLE patients, researchers and clinicians should be cautious in interpreting PDQ-20 results using the current four factors (subscales). Further validity analyses, including exploratory factor analyses, are needed.

\section{CS-23 REMISSION AND LOW DISEASE ACTIVITY STATE ARE ASSOCIATED WITH A BETTER HEALTH-RELATED QUALITY OF LIFE IN SYSTEMIC LUPUS ERYTHEMATOSUS IN A PRIMARILY MESTIZO POPULATION}

\footnotetext{
${ }^{1,2}$ Manuel F Ugarte-Gil ${ }^{*}$, ${ }^{1}$ Rocio V Gamboa-Cardenas, 'Mariela Medina-Chinchón, ${ }^{1}$ Francisco Zevallos, ${ }^{1}$ Cristina Reátegui-Sokolova, ${ }^{1,2}$ Claudia Elera-Fitzcarrald, ${ }^{1}$ Victor Pimentel-Quiroz, ${ }^{1}$ Jose Alfaro-Lozano, 1,3Zoila Rodriguez-Bellido, ${ }^{1,3}$ Cesar A PastorAsurza, ${ }^{4}$ Graciela S Alarcón, ${ }^{1,3}$ Risto Perich-Campos. ${ }^{1}$ Rheumatology Department. Hospital Guillermo Almenara Irigoyen. EsSalud; ${ }^{2}$ Universidad Cientifica del Sur; ${ }^{3}$ Universidad Nacional Mayor de San Marcos; ${ }^{4}$ School of Medicine, University of Alabama at Birmingham
}

\subsection{6/lupus-2018-Ism.58}

Background Achieving remission or low lupus disease activity state (LDAS) in systemic lupus erythematosus (SLE) patients improves their prognosis in terms of damage accrual. But, there is not enough information about their impact on healthrelated quality of life (HRQoL). The aim of these analyses is to evaluate the association between remission or LDAS and HRQoL, after adjustment for possible confounders.

Methods The Almenara Lupus Cohort was started in 2012; all patients evaluated at the Rheumatology Department were invited to participate. Visits were performed every six months. Socioeconomic and clinical data were recorded at every visit. Disease activity was ascertained with the Systemic Lupus Erythematosus Disease Activity Index 2000 (SLEDAI-2K), disease damage with the SLICC/ACR Damage Index (SDI) and HRQoL was measured with the LupusQoL. For theses analyses, data from the baseline visit were included. Remission was defined as a SLEDAI-2K=0, prednisone $\leq 5 \mathrm{mg} / \mathrm{d}$, immunosuppressants on maintenance dose, LDAS was defined as not on remission and a SLEDAI- $2 \mathrm{~K} \leq 4$, prednisone $\leq 7.5 \mathrm{mg} / \mathrm{d}$, immunosuppressants on maintenance dose. Univariable and multivariable lineal regression models, adjusted by age at diagnosis, disease duration, socioeconomic status, antimalarial use, disease duration, time of exposure to prednisone, damage and comorbidities were performed. Due to the relatively small number of patients on remission, remission and LDAS were analyzed together.

Results Two hundred and eighty patients were included, 259 $(92.5 \%)$ were female, mean age at diagnosis was 35.4 $(\mathrm{SD}=13.5)$ years, disease duration was $7.2(6.5)$ years. Fortyfive $(16.1 \%)$ were on remission, 94 (33.6\%) were on LDAS. Being on remission or LDAS was associated with a better HRQoL in the following domains, independently of possible confounders: physical health, planning, emotional health and body image. Univariable and multivariable analyses are depicted in table 1 .
Conclusions Being on remission or LDAS is associated with a better HRQoL independently of possible confounders.

\begin{tabular}{|c|c|c|c|c|}
\hline & Univariable & & Multivariable & \\
\hline & B (CI95\%) & $p$ value & B (CI95\%) & $p$ value \\
\hline Physical Health & $8.68(3.01 ; 14.34)$ & 0.003 & $6.80(1.78 ; 11.82)$ & 0.008 \\
\hline Emotional Health & $6.22(0.71 ; 11.72)$ & 0.027 & $5.76(0.33 ; 11.19)$ & 0.038 \\
\hline Body image & $9.84(4.52 ; 15.17)$ & $<0.001$ & $9.31(4.03 ; 14.58)$ & 0.001 \\
\hline Pain & $6.74(0.83 ; 12.64)$ & 0.025 & $5.49(-0.10 ; 11.09)$ & 0.054 \\
\hline Planning & $9.70(2.91 ; 16.50)$ & 0.005 & $7.89(1.33 ; 14.42)$ & 0.018 \\
\hline Fatigue & $3.05(-3.15 ; 9.26)$ & 0.335 & $2.57(-3.55 ; 8.69)$ & 0.410 \\
\hline Intimate relationship & $6.09(-2.94 ; 15.13)$ & 0.186 & $5.11(-3.13 ; 13.36)$ & 0.224 \\
\hline Burden to others & $6.91(-0.18 ; 14.00)$ & 0.056 & $6.13(-0.91 ; 13.16)$ & 0.088 \\
\hline
\end{tabular}

\section{CS-24 ASSOCIATION OF LIPOPROTEIN SUBFRACTIONS AND GLYCOPROTEIN ACETYLATION WITH CORONARY PLAQUE BURDEN IN SYSTEMIC LUPUS ERYTHEMATOSUS}

Monica M Purmalek, Philip M Carlucci, Amit K Dey, Maureen Sampson, Yenealem Temesgen-Oyelakin, Simantini Sakhardande, Joseph B Lerman, Alice Fike, Michael Davis, Jonathan H Chung, Taufiq Salahuddin, Zerai Manna, Sarthak Gupta, Marcus Y Chen, Sarfaraz Hasni, Nehal N Mehta, Alan T Remaley, Mariana J Kaplan*. National Institute of Arthritis and Musculoskeletal and Skin Diseases (NIAMS) and National Heart, Lung and Blood Institute (NHLBI), National Institutes of Health, Bethesda, MD

\subsection{6/lupus-2018-Ism.59}

Background Subjects with Systemic Lupus Erythematosus (SLE) display an increased risk of atherosclerotic cardiovascular disease (CVD) that is not explained by Framingham Risk. This study sought to investigate the utility of nuclear magnetic resonance (NMR) spectroscopy measurements of serum lipoprotein particle counts and size and glycoprotein acetylation (GlycA) to predict coronary atherosclerosis in SLE.

Methods Coronary plaque burden was assessed in SLE subjects and healthy controls using coronary CT angiography. Lipoproteins and GlycA were quantified by NMR spectroscopy.

Results SLE subjects displayed statistically significant decreases in high-density lipoprotein (HDL) particle counts and increased very low density lipoprotein (VLDL) particle counts compared to controls. Non-calcified coronary plaque burden (NCB) negatively associated with HDL subsets, whereas it positively associated with VLDL particle counts in multivariate adjusted models. GlycA was significantly increased in SLE sera compared to controls. In contrast to high-sensitivity C-reactive protein, elevations in GlycA in SLE significantly associated with NCB and insulin resistance.

Conclusions SLE patients display a proatherogenic lipoprotein profile that may significantly contribute to the development of premature CVD. The results demonstrate that NMR measures of GlycA and lipoprotein profiles, beyond what is captured in routine clinical laboratory tests for lipids, could be a useful tool in assessing CVD risk in SLE patients. 